\title{
Modelagem do volume de eucalipto em sistema de integração Lavoura- Pecuária-Floresta
}

\author{
Clebson Lima Cerqueira ${ }^{1 *}$ Rômulo Môra ${ }^{2}$ Helio Tonini ${ }^{3}$ Julio Eduardo Arce ${ }^{1}$ Gerson Dos Santos \\ Lisboa ${ }^{4}$ Carlos Cesar Cavassin Diniz ${ }^{3}$ Samuel de Pádua Carvalho ${ }^{4}$
}

\author{
${ }^{1}$ Universidade Federal do Paraná, Av. Pref. Lothário Meissner, 632, Jardim Botânico, Campus III, CEP 80210-170, Curitiba, PR, Brasil \\ ${ }^{2}$ Universidade Federal do Mato Grosso, Av. Fernando Corrêa da Costa, 2367, Boa Esperança, CEP 78060-900, Cuiabá, MT, Brasil \\ ${ }^{3}$ Empresa Brasileira de pesquisa Agropecuária, EMBRAPA Agrossilvipastoril, Rodovia dos Pioneiros MT 222 Km 2,5 s/n, CEP 78550-970, \\ Sinop, MT, Brasil; \\ ${ }^{4}$ Universidade Federal do Sul da Bahia , Rodovia de Acesso para Itabuna, km 39 - Ferradas, CEP 45613-204, Itabuna, BA Brasil
}

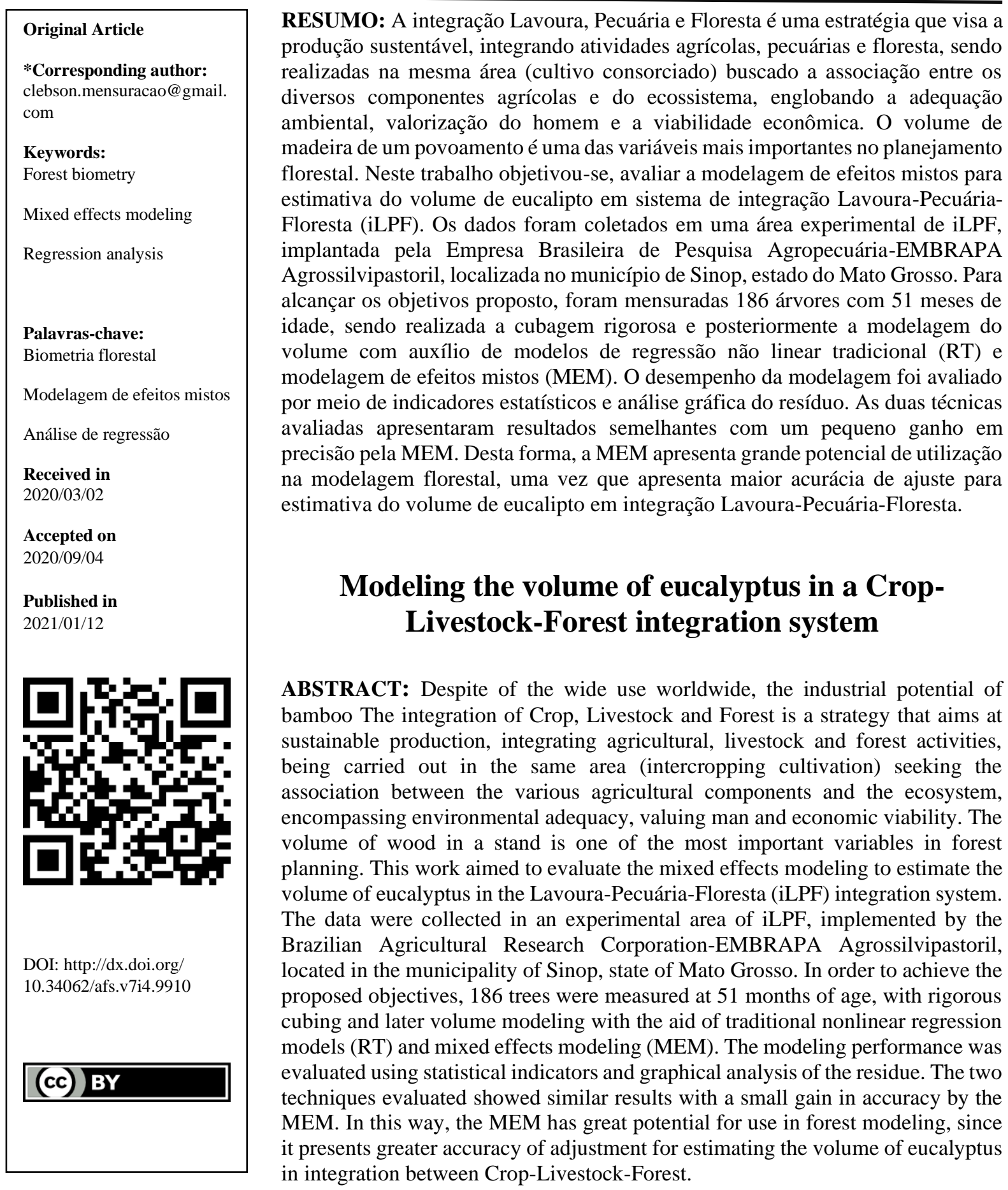




\section{Introdução}

O Brasil é considerado uma referência mundial na produção de grãos, proteína animal e produtos derivados das florestas plantadas. A produção significativa no país está relacionada à crescente demanda pelos diversos produtos de origem agropecuário e florestal. Desta forma, diferentes alternativas de cultivo que visam uma maior produtividade estão sendo sugeridas, tais como os sistemas de integração Lavoura-PecuáriaFloresta (iLPF).

Nos sistemas de iLPF, culturas agrícolas, árvores, pastagens e animais são integrados visando potencializar as interações ecológicas e econômicas (Torres et al. 2016). Esses sistemas de produção vêm crescendo por meio de programas de fomento, como o Programa Agricultura de Baixo Carbono (ABC), implantado pela Lei ${ }^{\circ} 12.187$ de 29 de dezembro de 2009, que institui a Política Nacional sobre Mudanças do Clima (PNMC).

Apesar do interesse e incentivo à adoção do sistema iLPF, ainda existem algumas lacunas no conhecimento a serem pesquisadas, a fim de subsidiar a elaboração, o planejamento e a adoção desses sistemas (Salles et al. 2012). Com isso, faz-se necessário o desenvolvimento de pesquisas visando a quantificação da produção, uma vez que essas informações subsidiam o planejamento florestal. Para isso, técnicas de modelagem por meio de estatísticas e ferramentas computacionais são utilizadas para se obter estimativas precisas da produção.

Para o gerenciamento dos empreendimentos florestais é fundamental quantificar e prognosticar com confiabilidade o estoque de madeira. Contudo, a dificuldade na determinação direta do volume das árvores se encontra na cubagem, sobretudo do ponto de vista econômico. Assim, faz-se necessário a utilização de modelos estatísticos para estimativa volumétrica, sendo que o volume é uma variável dependente e o diâmetro e altura são as variáveis independentes (Machado et al. 2002).

Com o advento dos recursos computacionais é possível a utilização de técnicas estatísticas mais atuais em diversas áreas da pesquisa científica. Dentre elas destaca-se a modelagem de efeitos mistos (MEM) com resultados promissores para estimativa do volume de árvores, como comprovado nos trabalhos de Packalén et al. (2011) e Vismara et al. (2016).

A MEM é caracterizada por utilizar em modelos lineares ou não lineares, parâmetros de efeitos fixos e aleatórios quando se tem medidas repetidas ou dados agrupados. Segundo Schabenberger e Pierce (2002) essa técnica deve ser utilizada quando se dispõe de um conjunto de dados que estão agrupados ou que necessita da inclusão de efeitos aleatórios.
Mesmo diante do crescente interesse pelo sistema de iLPF, informações biométricas das espécies que compõem esses sistemas ainda se encontra de forma incipiente. Desta forma, objetivou-se avaliar a modelagem de efeitos mistos para estimativa do volume de eucalipto em iLPF, sob a hipótese de que os modelos mistos proporcionam estimativas mais precisas quando comparado a regressão tradicional com efeitos fixos.

\section{Material e Métodos \\ Caracterização da área de estudo}

Os dados para realização deste estudo foram obtidos em duas áreas experimentais de sistema de integração Lavoura-Pecuária-Floresta (iLPF), denominadas como iLPF leite e iLPF corte, cuja implantação foi realizada pela Empresa Brasileira de Pesquisa Agropecuária (Embrapa Agrossilvipastoril) no município de Sinop, região norte de Mato Grosso.

De acordo com a classificação de Köppen, o clima da região é do tipo tropical de monção, com três meses de seca e precipitação pluviométrica acumulada no ano entre 2.500 e $2.800 \mathrm{~mm}$, temperatura média anual aproximada de 24 a $26{ }^{\circ} \mathrm{C}$, temperaturas máximas de $40{ }^{\circ} \mathrm{C}$ e mínimas de $20^{\circ} \mathrm{C}$, com altitude de $380 \mathrm{~m}$ (Alvares et al. 2013), além de relevo plano e predomínio do Latossolo VermelhoAmarelo Argiloso (Sema-MT, 2014).

A área experimental foi dividida em dois experimentos com mesmas características de solo e relevo, implantados em delineamento de blocos casualizados (DBC) com quatro tratamentos: Lavoura-Pecuária-Floresta (T1), Lavoura-PecuáriaFloresta (T2), Pecuária-Floresta (T3) e plantio homogêneo com eucalipto (T4).

$\mathrm{Na}$ iLPF leite foi instalado o tratamento T1, com arranjo das árvores em renques duplos e T2 as árvores foram estabelecidas em faixas com três linhas de plantio, consorciados (T1 e T2) com pastagem de capim-piatã (Brachiaria brizantha cv. Piatã) em integração com milho para silagem e criação de gado leiteiro girolanda. Na iLPF corte foi instalado $\mathrm{o}$ tratamento $\mathrm{T} 3 \mathrm{com}$ as árvores estabelecidas em faixas com três linhas de plantio, consorciados com capim-piatã (Brachiaria brizantha cv. Piatã) e criação de boi nelore para corte. O T4 foi composto apenas pelo plantio homogêneo de eucalipto.

Os tratamentos foram estabelecidos em diferentes arranjos de integração (Figura 1) e (Tabela 1). O componente florestal utilizado foi um clone do híbrido Eucalyptus grandis $x$ Eucalyptus urophylla e a orientação de plantio das árvores nas linhas foi no sentido leste oeste. 


\section{Cerqueira et al.}

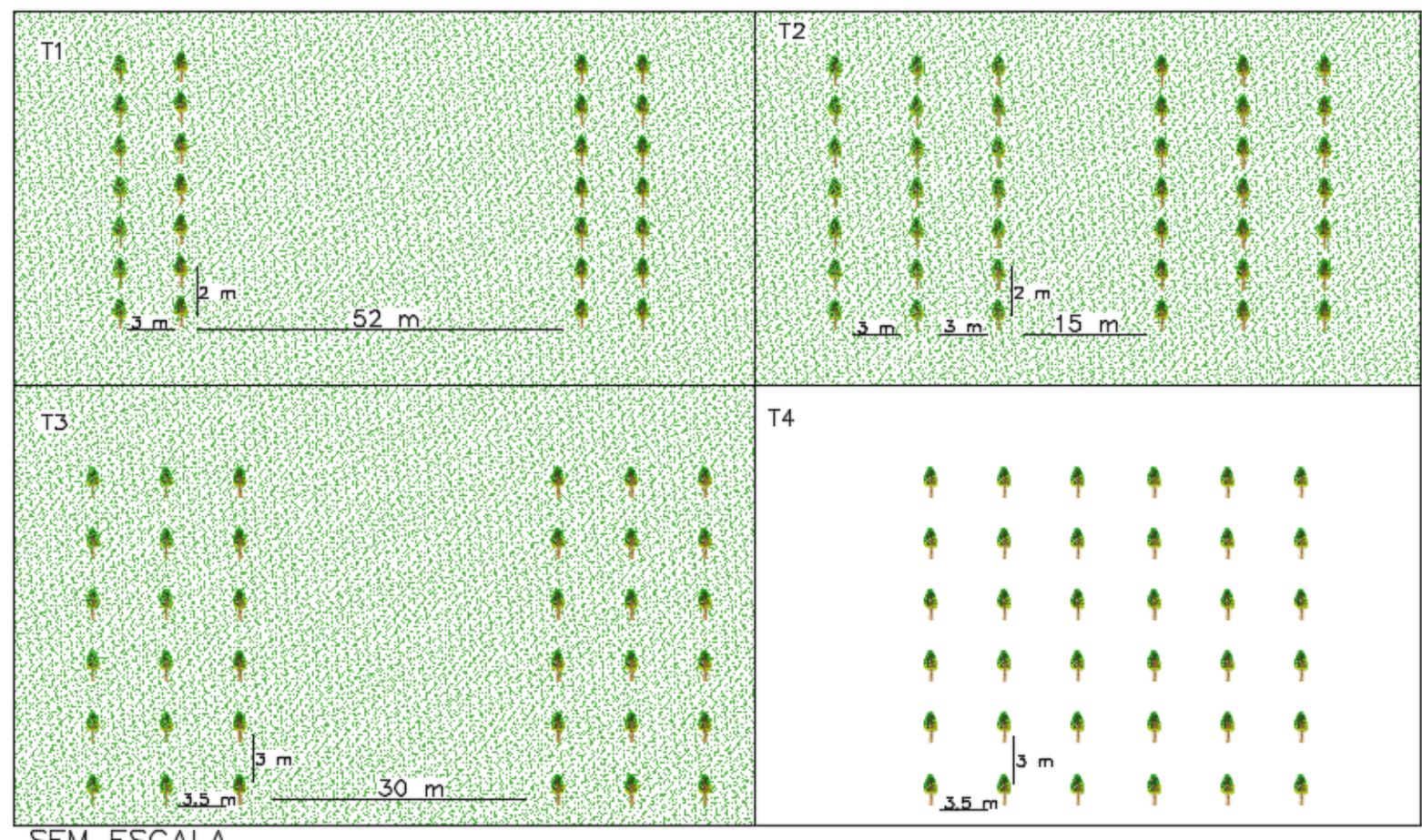

SEM ESCALA

Figura 1. Distribuição das árvores nos diferentes tratamentos avaliados.

\section{Base de dados}

Para obtenção dos dados foi realizado inventário florestal com estabelecimento de parcelas permanentes com dimensão de $882 \mathrm{~m}^{2}$ na linha central de cada tratamento (T1, T2 e T3) e no centro da área do monocultivo (T4), totalizando em 81 árvores mensuradas em cada parcela.

Os dados foram obtidos por meio da cubagem de 186 árvores aos 51 meses de idade distribuídas entre os tratamentos estudados (Tabela 1).

Tabela 1. Árvores cubadas nos diferentes tratamentos do sistema de integração Lavoura-Pecuária-Floresta.

\begin{tabular}{cccccccc}
\hline Tratamentos & Arranjo espacial (m) & $\mathrm{N}($ árv.ha-1) & $\mathrm{n}$ & $\begin{array}{c}\text { Amp./dap } \\
(\mathrm{cm})\end{array}$ & $\mathrm{L}$ & $\mathrm{IMA}$ & $\mathrm{Vt}\left(\mathrm{m}^{3}\right)$ \\
\hline $\mathrm{T} 1$ & $3 \times 2 \times 52$ & 189 & 21 & $5,3-20,8$ & 2 & 9,13 & 40,61 \\
$\mathrm{~T} 2$ & $3 \times 2 \times 15$ & 714 & 56 & $5-19,4$ & 3 & 28,1 & 124,1 \\
$\mathrm{~T} 3$ & $3,5 \times 3 \times 30$ & 270 & 54 & $9,4-23,7$ & 3 & 7,9 & 36,9 \\
T4 & $3,5 \times 3$ & 952 & 55 & $8,6-18,6$ & - & 31,2 & 145,7 \\
\hline Total & & 2125 & 186 & & & &
\end{tabular}

N (árv.ha-1): número de árvores por hectare; árv: árvores; n: número de árvores cubadas; Amp./dap: amplitude dos diâmetros; L: número de linhas de plantio com árvores nos tratamentos (T1,T2 e T3); IMA: incremento médio anual por tratamento; $\mathrm{V}$ t: volume total por tratamento.

A seleção das árvores para cubagem foi realizada por meio da distribuição diamétrica das árvores para cada tratamento, provenientes dos dados do inventário florestal, com a definição de 10 classes diamétricas e 5 árvores cubadas por classe nos tratamentos (T2, T3 e T4). No tratamento (T1) devido as árvores das duas menores classes serem pouco representativas, suprimidas e na sua grande maioria mortas, foram definidas 8 classes e amostradas três árvores por classe diamétrica.
As árvores selecionadas foram abatidas, seccionadas e os diâmetros com casca foram mensurados com auxílio da suta e trena nas seguintes alturas: $0,10 \mathrm{~m} ; 0,70 \mathrm{~m} ; 1,30 \mathrm{~m}$ e posterior em intervalos de $1,0 \mathrm{~m}$ até um diâmetro mínimo de aproximadamente $1,0 \mathrm{~cm}$. Em cada árvore mensurou-se, além dos diâmetros (di) nas várias alturas (hi), o diâmetro a 1,30 m do nível do solo (d) e a altura total (h). A obtenção do volume individual 
das árvores, considerado como real, foi determinado pela metodologia de Smalian.

\section{Modelagem do volume}

Para estimativa do volume foram empregadas duas técnicas de modelagem: regressão tradicional (regressão não linear) (RT) com todos os parâmetros fixos comum a todos as árvores e modelagem de efeitos mistos (MEM) com parâmetros específicos por grupos de árvores.

O modelo de regressão utilizado com a RT e MEM foi proposto por Schumacher e Hall (1933) (Equação 1). Utilizou-se este modelo por ser um dos mais difundido no setor florestal por suas propriedades estatísticas, uma vez que fornecem quase sempre estimativas acuradas e não tendenciosas (Campos; Leite, 2013).

$$
V t=\emptyset_{0} * d^{\emptyset_{1}} * h^{\emptyset_{2}}+\varepsilon
$$

Em que: $\mathrm{Vt}=$ volume total; $\mathrm{h}=$ altura total $(\mathrm{m}) ; \mathrm{d}=$ diâmetro a $1,30 \mathrm{~m}$ de altura $(\mathrm{cm}) ; \emptyset_{i}=$ coeficientes; $\varepsilon=$ erro aleatório.

A estrutura do modelo com efeitos mistos para estimativa do volume está representada na Equação 2.

$$
\emptyset=\left[\begin{array}{l}
\emptyset_{0} \\
\emptyset_{1} \\
\emptyset_{2}
\end{array}\right]=\left[\begin{array}{l}
\beta_{0} \\
\beta_{1} \\
\beta_{2}
\end{array}\right]+\left[\begin{array}{l}
b_{0} \\
b_{1} \\
\beta_{2}
\end{array}\right]=\beta+\mathrm{b}
$$

em que: $\mathrm{b} \sim \mathrm{N}\left(0, \sigma^{2}\right)$; e $\varepsilon \sim \mathrm{N}\left(0, \sigma^{2} \mathrm{I}\right)$. O vetor $\beta$ representa os efeitos fixos e o vetor $b$ os efeitos aleatórios.

O ajuste do modelo misto foi realizado de forma não linear, aplicando a modelagem não linear mista (nlme) adotando como efeito aleatório os diferentes tratamentos, com a finalidade de aumentar a precisão das predições de volume. A técnica foi aplicada também com objetivo de agrupar os dados obtidos nos diferentes espaçamentos e arranjos de integração, buscando possíveis melhorias nas estimativas.

Os ajustes foram realizados no Software R, por meio do pacote $n l m e$ desenvolvido por Pinheiro e Bates (2000). A função nlme do pacote nlme estima os parâmetros de efeitos fixos e os parâmetros de covariância associados aos parâmetros de efeitos aleatórios.

Tabela 2. Coeficientes e estatística de precisão para estimativa do volume nos diferentes tratamentos de integração Lavoura-Pecuária-Floresta.

\begin{tabular}{cccccc}
\hline Trat. & $\emptyset_{\mathbf{0}}$ & $\emptyset_{\mathbf{1}}$ & $\emptyset_{2}$ & $\mathbf{r}_{\mathbf{y y}}(\%)$ & RQEM (\%) \\
\hline T1 & $0,00009^{*}$ & $2,07000^{*}$ & $0,56900^{*}$ & 97,902 & 9,02 \\
T2 & $0,00005^{*}$ & $1,69900^{*}$ & $1,11000^{*}$ & 98,013 & 9,08 \\
T3 & $0,00006^{*}$ & $1,57500^{*}$ & $1,15600^{*}$ & 98,200 & 4,51 \\
T4 & $0,00002^{*}$ & $1,77100^{*}$ & $1,33200^{*}$ & 98,262 & 4,88 \\
\hline
\end{tabular}

Trat $=$ Tratamentos; $\emptyset_{i}=$ coeficientes estimados; $*=$ significativo.
A técnica nlme possibilita implementar um processo de dois estágios para a estimativa dos parâmetros. Inicialmente é realizado o processo dos mínimos quadrados não lineares para estimativa dos parâmetros, posteriormente sucessivos ajustes são feitos com os procedimentos de estimação por modelagem mista linear em uma função de aproximação linear com base na expansão da série de Taylor até a convergência ser alcançada. Mais detalhe sobre essa metodologia pode ser consultado no trabalho desenvolvido por Pinheiro e Bates (2000).

Os ajustes do modelo e procedimentos estatísticos foram realizados no Software R, versão 3.3 (R Development Core Team, 2016). Os ajustes pela RT foram realizados com a função $\mathrm{nls}$, pelo método interativo utilizando o algoritmo de GaussNewton. Após o ajuste foi avaliado a significância dos coeficientes das equações ajustadas por meio do teste $\mathrm{t}$ de Student. A precisão e acurácia da RT e MEM foi avaliada com base nos seguintes critérios estatísticos: maior coeficiente de correlação linear de Pearson $\left(\mathrm{r}_{\mathrm{yy}} \hat{)}\right.$, menor valor da raiz do erro quadrado médio percentual (RQEM \%). e análise gráfica dos resíduos em percentagem $(\mathrm{E} \%)$, calculados pelas equações 3,4 e 5 respectivamente.

$$
\begin{aligned}
& \mathrm{r}_{\mathrm{yy}}=\left(\frac{\left[\sum_{\mathrm{i}=1}^{\mathrm{n}} \mathrm{y}_{\mathrm{i}} \hat{\mathrm{y}}_{\mathrm{i}}-\frac{\left(\sum_{\mathrm{i}=1}^{\mathrm{n}} \hat{\mathrm{y}}_{\mathrm{i}}\right)\left(\sum_{\mathrm{i}=1}^{\mathrm{n}} \mathrm{y}_{\mathrm{i}}\right)}{\mathrm{n}}\right]^{2}}{\left[\sum_{\mathrm{i}=1}^{\mathrm{n}} \mathrm{y}_{\mathrm{i}}{ }^{2}-\frac{\left(\sum_{i=1}^{\mathrm{n}} \mathrm{y}_{\mathrm{i}}\right)^{2}}{\mathrm{n}}\right]\left[\sum_{\mathrm{i}=1}^{\mathrm{n}} \mathrm{y}_{\mathrm{i}}{ }^{2}-\frac{\left(\sum_{i=1}^{\mathrm{n}} \hat{\mathrm{y}}_{\mathrm{i}}{ }^{2}\right.}{\mathrm{n}}\right]}\right) \\
& \text { RQEM } \%=\frac{100}{\bar{y}_{i}} \sqrt{\frac{\sum_{i=1}^{n}\left(y_{i}-\hat{y}_{i}\right)^{2}}{n}} \\
& \mathrm{E} \%=\left(\frac{\mathrm{y}_{\mathrm{i}}-\hat{\mathrm{y}}_{\mathrm{i}}}{\mathrm{y}_{\mathrm{i}}}\right) * 100
\end{aligned}
$$

Em que: $\hat{y}_{\mathrm{i}}:=$ variável estimada; $\mathrm{y}_{\mathrm{i}}=$ variável observada; $\bar{y}_{\mathrm{i}}=$ média da variável observada; $\mathrm{n}=$ número de observações.

\section{Resultados}

O teste de significância dos coeficientes das equações apresentou resultados significativos $(\mathrm{p} \leq 0,05)$ em todos os tratamentos, indicando que as variáveis independentes explicam a variabilidade do volume das árvores (Tabela 2). 


\section{Cerqueira et al.}

As equações ajustadas apresentaram coeficiente de correlação r"y" "y" ^variando de 97 a 98,2 \% e erro quadrado médio percentual RQEM variando entre 4 e $9 \%$. Dentre os tratamentos estudados percebe-se um menor RQEM nas estimativas obtidas nos tratamentos T3 e T4.

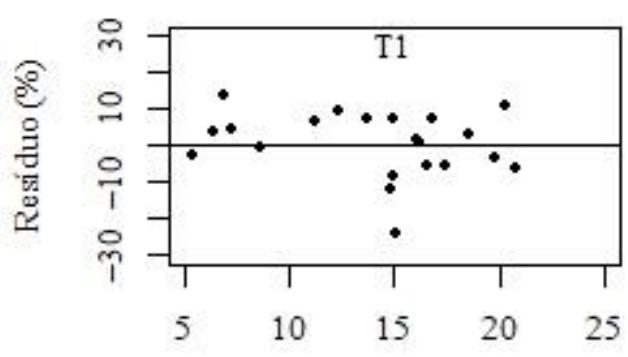

$\mathrm{d}(\mathrm{cm})$
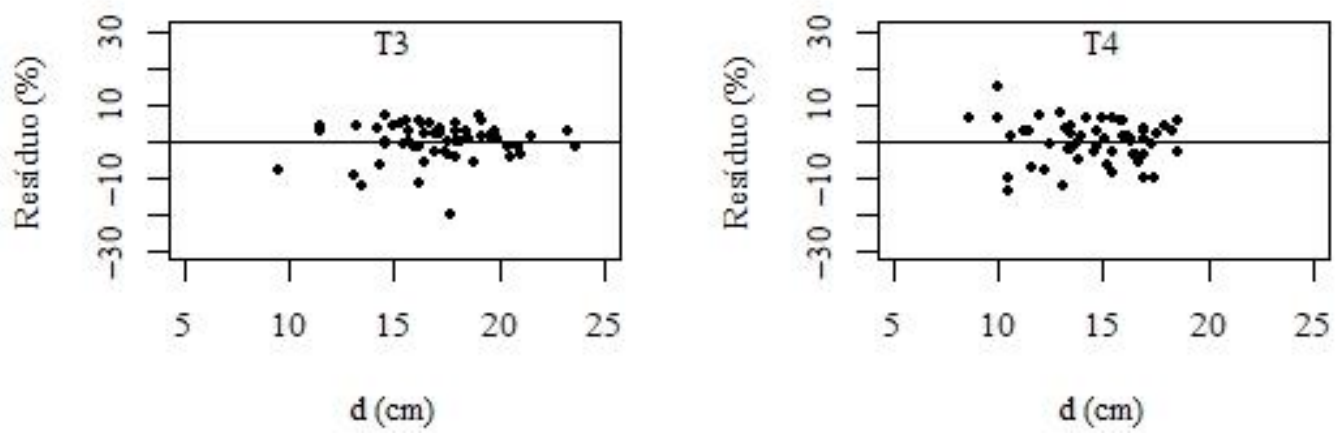

Figura 2. Distribuição dos resíduos em porcentagem para estimativa do volume nos diferentes tratamentos de integração Lavoura-Pecuária-Floresta.

Nota-se que os resíduos estão distribuídos aleatoriamente em torno de zero da linha de regressão, indicando boa precisão entre os valores observados e estimados.

Com base nas estatísticas de ajuste e análise gráfica, confirma-se a precisão do modelo de Schumacher e Hall para estimativa do volume de eucalipto em iLPF.
Para uma melhor avaliação da acurácia dos ajustes e para complementar os critérios estatísticos analisados, gerou-se os gráficos de resíduos para cada tratamento (Figuras 2).

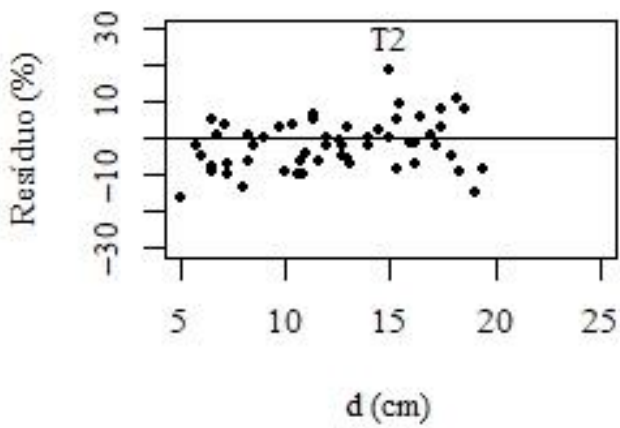

Tabela 3. Coeficientes aleatórios por tratamento e fixos para todo o conjunto de dados do sistema de integração Lavoura-Pecuária-Florestal.

\begin{tabular}{cccc}
\hline Trat. & \multicolumn{1}{c}{$\emptyset_{0}$} & $\emptyset_{1}$ & $\emptyset_{2}$ \\
\hline & \multicolumn{2}{c}{ Parâmetros de efeitos aleatórios } \\
T1 & $0,00005^{*}$ & 1,79782 & 1,01359 \\
T2 & $0,00005^{*}$ & 1,80744 & 1,00562 \\
T3 & $0,00005^{*}$ & 1,58052 & 1,19370 \\
T4 & $0,00005^{*}$ & 1,73270 & 1,06753 \\
\hline & & Parâmetros fixos & 1,0700 \\
\hline
\end{tabular}

Trat $=$ Tratamentos; $\mathrm{T}=$ Dados agrupados $; \emptyset_{i}=$ coeficientes estimados; $*$ = significativo

Adv. For. Sci, Cuiabá, v. 7, n. 4, p. 1213-1221, 2020 


\section{Cerqueira et al.}

As estatísticas de precisão apontam que a MEM e RT resultaram em estimativas acuradas, no entanto, nota-se que a MEM apresenta maior $\mathrm{r}_{\mathrm{yy}} .(\%)$ e menor RQEM (\%) (Tabela 4).

Tabela 4. Estatísticas de precisão da modelagem mista e regressão tradicional para estimativa do volume das árvores de eucalipto no sistema de integração Lavoura-Pecuária-Floresta.

Table 4. Predictive statistics of mixed modeling and traditional regression for estimating the volume of
Eucalyptus trees in the crop-livestock-forestry integration system.

\begin{tabular}{ccc}
\hline Ajuste & $\mathbf{r}_{\mathbf{y y}}(\boldsymbol{\%})$ & RQEM (\%) \\
\hline RT & 97,347 & 7,95 \\
\hline MEM & 98,089 & 6,74 \\
\hline
\end{tabular}

Além destas estatísticas (Tabela 4), a análise gráfica dos resíduos foi utilizada para comparar as duas técnicas utilizadas (Figura 3).
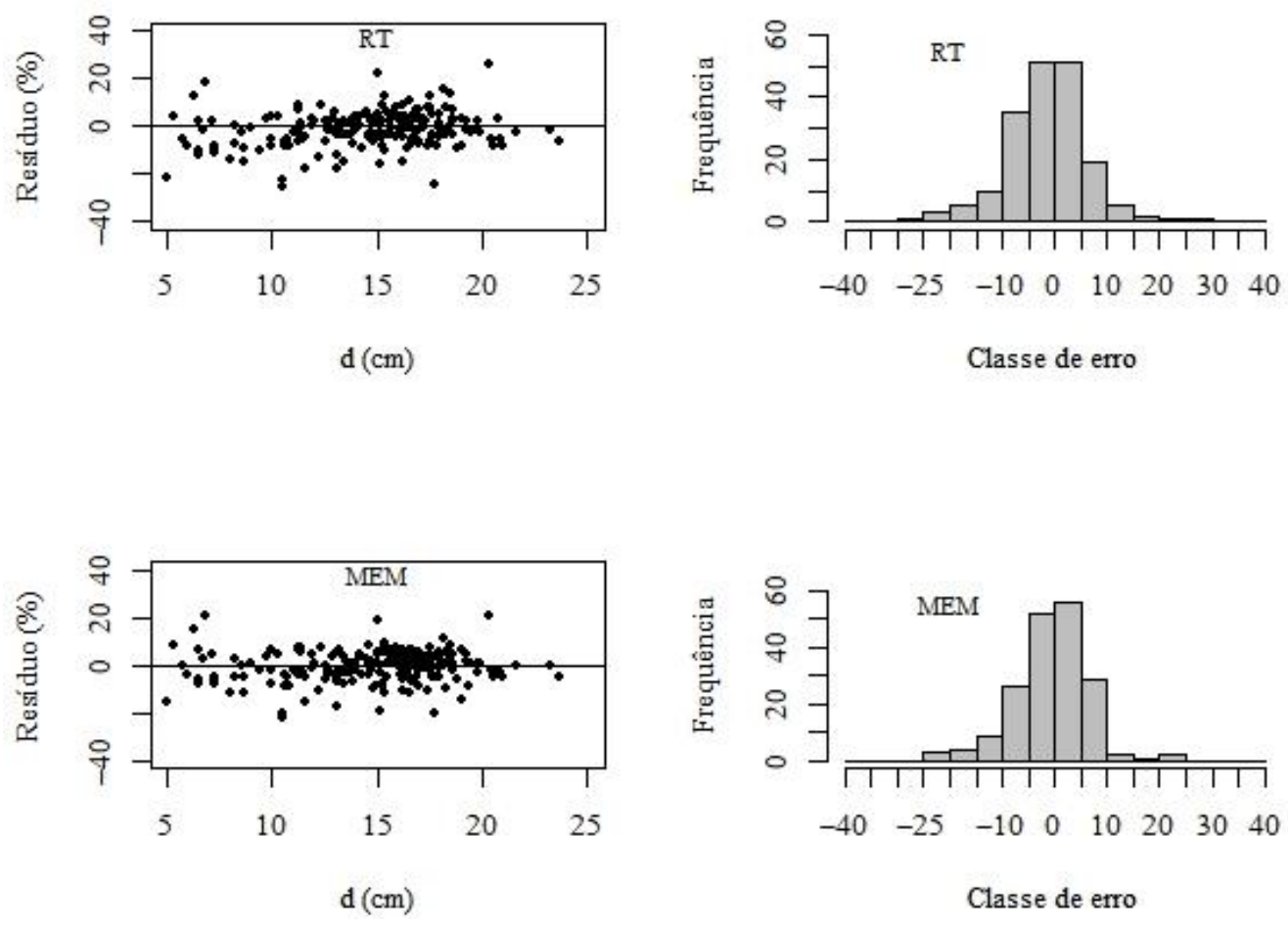

Figura 3. Distribuição dos resíduos em percentagem e histograma dos erros para a regressão tradicional (RT) e modelagem de efeitos mistos (MEM).

Os gráficos de resíduos permitem avaliar o comportamento dos erros entre as classes de diâmetro e observa-se similaridade entre RT e MEM, com distribuição adequada ao longo da linha de regressão, o que indica boa precisão entre os volumes observados e estimados. Entretanto, nota-se maior dispersão dos resíduos nas estimativas feitas pela RT. Os histogramas dos resíduos apresentaram distribuição simétrica e média em torno de 0 , confirmando a precisão das duas técnicas avaliadas.

Ao plotar os valores observados versus estimados (Figura 4), é possível notar que em ambas as técnicas a dispersão acontece ao longo da linha que parte da origem e forma um ângulo de 45 graus, indicando que os valores observados e estimados são coincidentes, contudo, ao considerar o coeficiente de determinação $\left(\mathrm{R}^{2}\right)$ da reta média, a MEM apresenta maior valor $(0,98)$ quando comparado ao da RT $(0,97)$.

Com base nas estatísticas de precisão, na distribuição residual, nos dados observados versus estimados, confirma-se que as duas técnicas apresentam resultados precisos para estimativa do volume das árvores amostradas, com pequena superioridade de precisão pela MEM. 


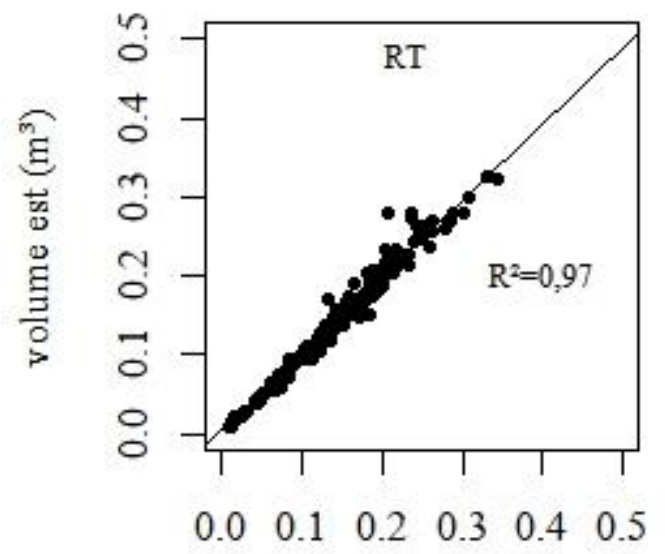

volume obs $\left(\mathrm{m}^{3}\right)$

Figura 4. Volume observado e estimado pela RT e MEM.

\section{Discussão}

Os indicadores estatísticos de acurácia assemelham-se a outros estudos de modelagem volumétrica para eucalipto (Azevedo et al. 2011; Leal et al. 2015) e, portanto, são considerados satisfatórios na modelagem para estimativa do volume de floresta plantada, sobretudo quando os valores de $\mathrm{r}_{\mathrm{yy}}$ aproxima-se de $99 \%$ e RQEM inferiores a $10 \%$.

A eficiência do modelo de Schumacher e Hall para predição do volume já foi comprovada e relatada por outros autores, dentre eles Müller et al. (2014), testando modelos volumétricos para estimativa do volume de eucalipto estabelecidos em sistema silvipastoril encontrou resultados precisos.

O modelo sugerido já é consagrado na ciência florestal pelo seu alto potencial nas predições de volume, corroborando com vários outros estudos que relatam a sua eficiência, afirmando ainda que esse modelo é um dos mais difundidos no meio florestal para expressar o volume de madeira em função do diâmetro e da altura total ou comercial, uma vez que resulta em estimativas acuradas (Campos e Leite, 2013).

O volume dos povoamentos florestais é influenciado por diversos fatores, dentre eles o espaçamento (Scolforo, 2006), desta forma, ao considerá-lo como efeito aleatório da MEM foi possível obter estimativas mais precisas.

O número de parâmetros aleatórios utilizados na MEM apresenta relação direta com a precisão das estimativas. Contudo, quando o modelo possui elevada quantidade de parâmetros, dificulta o procedimento matricial a ser realizado pelo Software e, em muitos casos não há convergência, portanto, nesses casos, o ideal é diminuir o número de parâmetros aleatórios do modelo.

O ganho em precisão proporcionado pela MEM já foi comprovado e relatado por outros autores, dentre outros, Packalén et al. (2011) com o

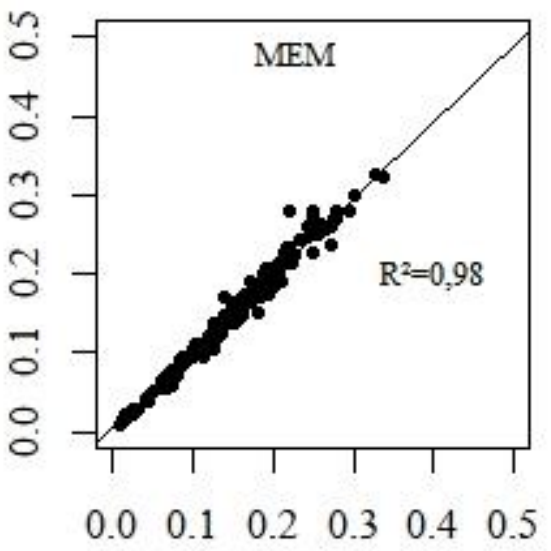

volume obs $\left(\mathrm{m}^{3}\right)$

emprego de modelos mistos para estimativa do volume de diferentes clones de eucalipto, concluíram que efeito aleatório do clone melhora consideravelmente a precisão. Gouveia et al. (2015) ajustaram o modelo de Schumacher e Hall com efeitos mistos, inserindo como efeito aleatório a própria árvore e concluíram que os modelos mistos são eficazes na modelagem do volume de eucalipto, recomendando seu uso para tal finalidade. Vismara et al. (2016), avaliou o modelo de Schumacher e Hall com efeitos mistos para estimativa do volume de Eucalyptus grandis em diferentes rotações e fazendas, concluíram que o modelo de efeitos mistos no nível fazenda pode melhorar as predições de volume em plantações de eucaliptos.

Os resultados obtidos permitem ressaltar a importância da modelagem que inclui efeitos aleatórios em relação aos modelos de efeitos fixos. Gouveia et al. (2015) com a aplicação de modelos mistos para estimativa do volume de eucalipto, ressaltou que uma das vantagens é a redução do erro padrão residual.

Com os resultados obtidos, certifica-se que apesar da RT proporcinar estimativas precisas do volume de eucalipto, como comprovado por Azevedo et al. (2011); Müller et al. (2014), a utilização de técnicas sofisticadas com o emprego de ferramentas computacionais surge como uma alternativa para aprimorar o processamento de dados e estimar com confiabilidade a produção florestal. Carvalho et al. (2011) acrescenta que os recursos computacionais são de grande relevância para os processos de modelagem e evolução técnicocientífico da mensuração florestal.

Cabe salientar, que além das estimativas acuradas, outro fator importante da MEM é a possibilidade de agrupar dados de condições florestais diferentes (espaçamentos, material genético, sítio, classe diamétrica e idade) e realizar o procedimento de ajuste com uma única base de dados reduzindo o número de equações com ganhos 
de tempo e acurácia, e sobretudo, isso reflete nos custos do inventário florestal. Além disso, a MEM permite trabalhar com conjunto de dados que não atendem a alguns pressupostos da regressão, como: independência entre as observações, distribuição aleatória, normal e idêntica dos resíduos, com média zero e variância constante (Lappi, 1991).

Com isso, nota-se a importância de estudos com aplicação da MEM para predição do volume das árvores. Outro fator de grande relevância é a possibilidade de redução do número de árvores amostra para os ajustes, pois conforme Dorado et al. (2006) e Mendonça et al. (2015) o emprego da teoria dos modelos mistos possibilita a redução da intensidade amostral necessária para ajuste de modelos com precisão adequada.

A redução da intensidade amostral é de suma importância para a mensuração florestal e principalmente quando se trabalha com sistemas iLPF, tendo em vista que nesses há uma menor quantidade de árvores quando comparado aos sistemas convencionais de produção. Desta forma, a MEM é uma ferramenta promissora para estimativa do volume de madeira das árvores, podendo ser utilizadas para dar suporte ao manejo florestal de eucalipto em sistema de iLPF.

\section{Conclusões}

O volume de árvores de Eucalyptus grandis, pode ser obtido com precisão, utilizando modelos matemáticos, os quais são ferramentas eficientes no meio florestal para a quantificação volumétrica.

O modelo de Schumacher e Hall é adequado para estimativa do volume das árvores de eucalipto em diferentes arranjos de plantio e espaçamentos.

A modelagem de efeitos mistos apresenta grande potencial de utilização em modelagem florestal, uma vez que apresenta maior acurácia de ajuste para estimativa do volume de eucalipto em integração Lavoura-Pecuária-Floresta.

\section{Agradecimentos}

Os autores agradecem à Embrapa-Agrossilvipastoril e ao Conselho Nacional de Desenvolvimento Científico e Tecnológico (CNPQ).

\section{Referências bibliográficas}

Alvares CA, Stape JL, Sentelhas PC, Gonçalves JLM, Sparovek G (2013) Köppen's climate classification map for Brazil. Meteorologische Zeitschrift, 22(6): p.711-728. doi:10.1127/09412948/2013/0507.

Azevedo GB, Sousa GTO, Barreto PAB, Conceição Júnior V 2011 Estimativas volumétricas em povoamentos de eucalipto sob regime de alto fuste e talhadia no sudoeste da Bahia. Pesquisa Florestal Brasileira, 31 (68): p. 309-318. DOI: 10.1127/09412948/2013/0507.
Campos JCC, Leite HG 2013 Mensuração florestal: perguntas e respostas. Viçosa: UFV, 4. Ed. 605p.

Carvalho SPC, Calegario N, Silva FF, Borges LAAC, Mendonça AR, Lima MP (2011) Modelos não lineares generalizados aplicados na predição da área basal e volume de Eucalyptus clonal. Cerne, Lavras, 17(4): p. 541 - 548. DOI: https://doi.org/10.1590/S010477602011000400013.

Dorado FC, Diéguez-Aranda U, Anta MB, Rodriguez MS, Von Gadow K (2006) A generalized height-diameter model including random components for radiata pine plantations in northwestern Spain. Forest Ecology and Management, 229: p. 202-213. DOI:org/10.1016/j.foreco.2006.04.028.

Gouveia JF, Silva JAA, Ferreira RLC, Gadelha FHL, Filho LMAL (2015) Modelos volumétricos em clones de Eucalyptus no polo gesseiro do Araripe, Pernambuco. Floresta, 45 (3): p.587-598. DOI: 10.5380/rf.v45i3.36844.

Lappi J (1991) Calibration of height and volume equations with random parameters. Forest Science, 37 (3): p.781-801.

Leal FA, Cabacinha CD, Castro RVO, Matricardi EAT (2015) Amostragem de árvores de Eucalyptus na cubagem rigorosa para estimativa de modelos volumétricos. Revista Brasileira de Biometria, 33(1): p. 91-103.

Machado AS, Conceição MB, Figueiredo Filho A (2002) A modelagem do volume individual para idades e regimes de desbaste em plantações de Pinus oocarpa. Ciencias Exatas e Naturais, 4(2):p. 41-50.

Mendonça AR, Carvalho SPC, Calegario N (2015) Modelos hipsométricos generalizados mistos na predição da altura de Eucalyptus sp. Cerne, 21(1):p. 107-105. DOI: 10.1590/01047760201521011191.

Müller MD, Salles TT, Paciullo DSC, Brighenti AM, Castro CRT (2014) Equações de altura, volume e afilamento para eucalipto e acácia estabelecidos em sistema silvipastoril. Floresta, 44(3): p. 473-484

Packalén P, Mehtätalo L, Maltamo M (2011) ALSbased estimation of plot volume and site index in a Eucalyptus plantation with a nonlinear mixed-effect model that accounts for the clone effect. Forest Science, 68: p.1085-1092. DOI: 10.1007/s13595011-0124-9. 


\section{Cerqueira et al.}

Pinheiro JC, Bates DM (2000) Mixed-effects models in $S$ and S-PLUS. New York: Springer-Verlag. 528 p.

R Core Team (2016) R: A language and environment for statistical computing. $\mathrm{R}$ Foundation for Statistical Computing, Vienna, Austria. Diponível em: https://www.R-project.org/.

Salles TT, Leite HG, Oliveira Neto SN, Soares CPB, Paiva HN, Santos FL (2012) Modelo de Clutter na modelagem de crescimento e produção de eucalipto em sistemas de integração lavoura-pecuária-floresta. Pesquisa Agropecuária Brasileira, 48: p. 253 - 260.

Schabenberger O, Pierce FJ (2002) Contemporary statistical models: for the plant and soil sciences. 730p.

Schumacher FX, Hall FS (1933) Logarithmic expression of timber-tree volume. Journal of Agricultural Research, 47(9):p. 719-734.

SEMA-MT, Secretaria de estado meio ambiente de Mato Grosso. Características do Mato Grosso (2014) Disponível em: <http://www.sema.mt.gov.br/>. Acesso: 30/12/2017.

Torres CMME, Oliveira AC, Pereira BLC, Jacovine LAG, Oliveira Neto SN, Carneiro ACO, Torres CMME (2016) Estimativas da produção e propriedades da madeira de eucalipto em Sistemas Agroflorestais. Scientia Forestalis 44(109): p. 137148. DOI: dx.doi.org/10.18671/scifor.v44n109.13.

Vismara ES, Mehtätalo L, Batista JLB (2016) Linear mixed-effects models and calibration applied to volume models in two rotations of Eucalyptus grandis plantations. Canadian Journal of Forest Research, 46(1):p.132-141. DOI: org/10.1139/cjfr2014-0435. 